\title{
Perturbations of the Quintom Models of Dark Energy and the Effects on Observations
}

\author{
Gong-Bo Zhao ${ }^{1}$, Jun-Qing Xia ${ }^{1}$, Mingzhe $\mathrm{Li}^{2}$, Bo Feng ${ }^{1}$, and Xinmin Zhang ${ }^{1}$ \\ 1 Institute of High Energy Physics, Chinese Academy of Sciences, \\ P.O. Box 918-4, Beijing 100049, People's Republic of China and \\ 2 Institut für Theoretische Physik, Philosophenweg 16, 69120 Heidelberg, Germany
}

\begin{abstract}
We study in this paper the perturbations of the quintom dark energy model and the effects of quintom perturbations on the current observations. Quintom describes a scenario of dark energy where the equation of state gets across the cosmological constant boundary $w=-1$ during evolution. We present a new method to show that the conventional dark energy models based on single k-essence field and perfect fluid cannot act as quintom due to the singularities and classical instabilities of perturbations around $w=-1$. One needs to add extra degrees of freedom for successful quintom model buildings. There are no singularities or classical instabilities in perturbations of realistic quintom models and they are potentially distinguishable from the cosmological constant. Basing on the realistic quintom models in this paper we provide one way to include the perturbations for dark energy models with parametrized equation of state across -1 . Compare with those assuming no dark energy perturbations, we find that the parameter space which allows the equation of state to get across -1 will be enlarged in general when including the perturbations.
\end{abstract}

\section{INTRODUCTION}

In 1998 two groups [1, 2] have independently discovered the accelerating expansion of our current universe based on the analysis of Type Ia Supernovae (SN) observations of the redshift-distance relations. In the framework of Friedmann-Robertson-Walker (FRW) cosmology, the acceleration has been attributed to the mysterious source dubbed dark energy. The simplest candidate for dark energy is a small positive cosmological constant, but it suffers from the difficulties associated with the fine tuning and the coincidence problem 3, 4]. The most popular alternative to the cosmological constant is the model of rolling scalar field-quintessence [5, 6]. In most cases the quintessence equation of state (EOS) $w$ changes slowly with time and can be well approximated with a constant $w$ with $w \geq-1$, 8, 8]. In the early probes of new physics, cosmologists have assumed a cosmological constant as the new component 1, 1, 9, 10, 11] and later fitted directly to the dynamical quintessence models [4, 12], or used a constant $w$ ], 8,13 , 14] where $w$ was restricted in the region of $w \geq-1$. In Ref. 15] the author firstly extended the fitting of dark energy to include $w<-1$ and found some mild preferences. The author constructed a toy model of rolling scalar field with a negative kinetic term and called it phantom [15]. The model of phantom has some theoretical problems [16] and there have been many attempts towards resolving them 17 .

The accumulation of the observational data $18,19,20,21,22,23$ has opened a robust window for probing the more detailed behaviors of dark energy. There have been many studies in reconstructing the evolution of its energy density [24] or equation of state [25, 26, 27] as a function of the redshift. Various parametrizations of $w$ as well as dark energy models have also been considered to fit directly to the observational data (e.g. 28, 29, 30, 31, 32, 33, 34, 35, 36, 37]). Based on the fact that current observations cannot exclude dark energy models with the equation of state getting across -1 during evolution with the redshift, we proposed a model dubbed quintom [35]. The model of quintom is a new scenario in the sense that the conventional quintessence or phantom models cannot realize the crossing of the cosmological boundary. Along this line the author in Ref. [38] has demonstrated that in the framework of general relativity the model of k-essence [39], where the scalar field of dark energy has non-canonical kinetic terms, cannot realize such a crossing behavior. A toy model with two rolling scalar fields which have opposite kinetic energy terms can easily realize the transition and it can be regarded as the simplest quintom model [35, 40]. Recently a single field quintom model was proposed in [41] by adding higher derivative operators in the Lagrangian. In the simplest case such a model is equivalent to the two-field case as proposed in 35]. In addition, the quintom model of dark energy are different from the quintessence or the phantom in the determination of the evolution and the fate of the universe. Due to its distinctive properties, the quintom model with oscillating equation of state across -1 can lead to the oscillations of the Hubble constant and a new scenairo of recurrent universe [42], which to some extent unifies the early inflation [43] and the current acceleration of the universe. Recently there have been a lot of interests in the phenomenological studies relevant to quintom models in the literature [44, 45, 46, 47, 48, 49, 50, 51, 52, 53, 54].

Current supernovae data alone, which make the only direct detection of dark energy, seem to favor a quintom-like model at around $2-\sigma$ level $31,32,35]$. The quintom model is also mildly favored in the combined analysis with the cosmic microwave background (CMB), large scale structure (LSS) and supernovae data [33, 34]. However, when some other observational data sets (such as the new observational data based on Chandra measurements of the X-ray gas mass fraction in $26 \mathrm{X}$-ray luminous galaxy clusters [23] or the recent new constraints from the bias and Ly $\alpha$ forest 
of the Sloan Digital Sky Survey (SDSS)) have been taken into account the situation changes and the preference for quintom-like dark energy models becomes weak [35, 36]. However the previous fittings in the literature on quintom-like dark energy models have either fully or partially neglected the perturbations, which in some sense do not describe the realistic models with EOS across -1 and will lead to some bias in the fittings. The aim of this paper is to develop a self-consistent way to include the perturbations of quintom in light of the observations. We will present a simple new method to show that conventional single perfect fluid and k-essence dark energy models cannot act as quintom, which is due to the singularities and classical instabilities of perturbations. Based on the realistic quintom models in this paper we will provide one way to include the perturbations for dark energy models with parametrized equation of state across -1 . Compared with those assuming no dark energy perturbations, we find that when including the perturbations the parameter space which allows the equation of state to get across -1 will be enlarged in general.

This paper is organized as follows: in section II we discuss the difficulty of quintom model buildings and provide a new proof regarding the impossibility of single perfect fluid and k-essence model as quintom, then present some viable quintom models; in section III we study in detail the perturbations of the quintom models; in section IV we investigate the possible signatures of quintom models of scalar fields and the effects of quintom perturbations on the observations; in section $\mathrm{V}$ we provide one way to include the perturbations for models of dark energy with a parametrized equation of state across -1 ; we conclude in section VI.

\section{QUINTOM MODEL BUILDING}

\section{A. Difficulties of Quintom Model Buildings}

We start with a brief overview on the arguments against the possibility of realizing the quintom scenario with a single fluid or a single scalar field in the conventional framework.

Consider firstly a single perfect fluid, the energy-momentum tensor has the conventional form,

$$
T_{\mu \nu}=-P g_{\mu \nu}+(\rho+P) u_{\mu} u_{\nu}
$$

where $\rho$ and $P$ are proper energy density and pressure, $u_{\mu}$ is 4 -velocity with $u^{\mu} u_{\mu}=1$. The energy density and the pressure of the fluid can be parametrized as [55]

$$
\begin{aligned}
\rho & =f(n), \\
P & =n f^{\prime}(n)-f(n),
\end{aligned}
$$

where $f(n)$ is a positive function of $n$. The introduced variable $n$ can be identified with the number density and the prime represents derivative with $n$. The equations of motion are just the covariant conservation equations of the momentum tensor, $\nabla_{\mu} T^{\mu \nu}=0$. In spatially flat FRW spacetime

$$
d s^{2}=a^{2}(\tau)\left(d \tau^{2}-d x^{i} d x_{i}\right)
$$

there is only one equation,

$$
\dot{\rho}+3 \mathcal{H}(\rho+P)=0
$$

where the dot is the derivative with the conformal time $\tau$ and $\mathcal{H}=\dot{a} / a$. Combining Eq.(4) with Eq. (2), we get

$$
f^{\prime}(n)(\dot{n}+3 \mathcal{H} n)=0
$$

Since $f^{\prime}(n)$ does not vanish everywhere (otherwise it corresponds to the cosmological constant), one has the conservation equation of the number density $\dot{n}+3 \mathcal{H} n=0$. In the expanding universe, $n$ will decrease monotonically with time.

In the following we will demonstrate that the system suffers from the problem of singularity and classical instability when the equation of state of the perfect fluid crosses the boundary of -1 . Let us assume that the system crosses -1 at the point of $n=n_{0} \neq 0$. At this point $\rho\left(n_{0}\right)+P\left(n_{0}\right)=0, f^{\prime}\left(n_{0}\right)=0$ and $f^{\prime}(n)$ will change the sign after the crossing. So, in the neighborhood of $n_{0}$, we can expand $f^{\prime}(n)$ in terms of $\left(n-n_{0}\right)$. The adiabatic sound speed square in this neighborhood is

$$
c_{s}^{2} \equiv \frac{d P}{d \rho}=\frac{n f^{\prime \prime}(n)}{f^{\prime}(n)} \simeq \frac{n}{n-n_{0}} .
$$


We can see that $c_{s}^{2}$ is singular at the crossing point. Moreover, $c_{s}^{2}$ is negative in the region of $n<n_{0}$. And when $n$ approaches $n_{0}$ from this side, it will approach $-\infty$. A negative $c_{s}^{2}$ will induce a serious classical instability to the system, the perturbations on small scales will increase quickly with time and the late time history of the structure formations will get significantly modified. This will inevitably lead to the fact that such models can never be compatible with the observations relevant to structure formations, such as CMB and LSS.

As shown above it is impossible to realize the quintom scenario with a single perfect fluid, now we turn to consider the model of scalar field. The general model of dark energy with a single scalar field and an arbitrary function of its first derivative in the Lagrangian was proposed in Ref. 39] and named as k-essence. Its Lagrangian usually has a non-canonical form

$$
\mathcal{L}=P(\phi, X)
$$

with

$$
X \equiv \frac{1}{2} \nabla_{\mu} \phi \nabla^{\mu} \phi .
$$

The energy-momentum tensor of this system has the same form as that of the single perfect fluid Eq. (1), where

$$
\begin{aligned}
& \rho=2 X P,_{X}-P, \\
& u_{\mu}=\frac{\nabla_{\mu} \phi}{\sqrt{2 X}} .
\end{aligned}
$$

Let's see under what conditions the system will be able to cross the barrier of $w=-1$. In order to do that, one requires $\rho+P$ to vanish at a point of $\left(\phi_{0}, X_{0}\right)$ and change the sign after the crossing. This can only be achieved by requiring $P, X\left(\phi_{0}, X_{0}\right)=0$ and $P,{ }_{X}$ has different signs before and after the crossing since $X$ cannot be negative. The covariant conservation law of the energy-momentum tensor gives the equation of motion,

$$
\left(P,,_{X} g^{\mu \nu}+P,_{X X} \nabla^{\mu} \phi \nabla^{\nu} \phi\right) \nabla_{\mu} \nabla_{\nu} \phi+\rho_{\phi}=0 .
$$

From this equation, we obtain the equation for the background field

$$
\rho, X\left[\ddot{\phi}+\left(3 c_{s k}^{2}-1\right) \mathcal{H} \dot{\phi}\right]+a^{2} \rho,_{\phi}=0,
$$

and the perturbation to the first order (neglecting the metric perturbations for the time being):

$$
\ddot{u}+\left[-c_{s k}^{2} \nabla^{2}-\ddot{z} / z-3 c_{s k}^{2}\left(\dot{\mathcal{H}}-\mathcal{H}^{2}\right)\right] u=0,
$$

where

$$
u \equiv a z \frac{\delta \phi}{\dot{\phi}}, \quad z \equiv \sqrt{\dot{\phi}^{2}|\rho, X|},
$$

and the effective sound speed is given by

$$
c_{s k}^{2} \equiv \frac{P, X}{\rho, X} .
$$

This sound speed $c_{s k}^{2}$ is often used in describing the perturbations of the scalar fields instead of the isentropic sound speed, which behave differently due to the intrinsic properties of the scalar fields [56]. For a conventional quintessence or phantom field, $c_{s k}^{2} \equiv 1$. The dispersion relation from Eq. (12) is

$$
\omega^{2}=c_{s k}^{2} k^{2}-\ddot{z} / z-3 c_{s k}^{2}\left(\dot{\mathcal{H}}-\mathcal{H}^{2}\right) .
$$

One of the conditions for the stability of k-essence perturbations is that $c_{s k}^{2}$ must be positive [39]. This requires that $\rho, X$ has the same behavior as that of $P, X$, i.e., it must vanish at the crossing point and change the sign after the crossing.

Similar to the analysis in the case of single fluid, we can see that $\ddot{z} / z$ diverges at the point $\left(\phi_{0}, X_{0}\right)$. This singularity is unavoidable in the perturbation equation and the physical quantities describing the fluctuations are not well defined. Generally, $\ddot{z}$ does not vanish at the crossing point, hence there exists a region in which $\omega^{2}<0$ and the perturbation is unstable. Furthermore, the canonical momentum defined by the Lagrangian (77) is

$$
\Pi=\frac{\partial P}{\partial \dot{\phi}}=P, X \frac{\dot{\phi}}{a^{2}} .
$$


Its derivative with respect to $\dot{\phi}$,

$$
\frac{\partial \Pi}{\partial \dot{\phi}}=\frac{\rho, X}{a^{2}}
$$

vanishes at the point of crossing. This shows that $\dot{\phi}$ is not a single valued function of the momentum $\Pi$ and we cannot get a canonical Hamiltonian transformed from the Lagrangian unambiguously [57]. The theory cannot be quantized in a canonical way. Hence we have shown that the conventional k-essence model cannot give rise to $w$ across -1 . A different proof is given in Ref. [38].

We should stress again that in realistic quintom model buildings one must consider the aspects of perturbations, where there are often dangerous instabilities in the conventional case. The concordance cosmology is based on the precise observations where many of them are tightly connected to the growth of perturbations and we must ensure the stability of perturbations. If we start with parametrizations of the scale factor [58] or EOS to construct quintom models, it can be realized arbitrarily if we do not consider the stability of perturbations. On the other hand when we start from scalar fields and use some phenomenological parametrizations it is in some sense very easy to resemble fluid behavior in the background evolutions. However the stability of perturbations must be considered.

\section{B. Some Viable Quintom Models}

As we demonstrated above in the conventional cases with a single fluid or a k-essence one cannot realize a viable model of quintom, we need to introduce extra degrees of freedom to realize the transition of $w$ across -1 . One of the possibilities is a system including two fluids with one being $w>-1$ and another $w<-1$. Specifically, consider a model which consists of two Chaplygin gases [59] with $P_{1}=-\lambda_{1} / \rho_{1}$ and $P_{2}=-\lambda_{2} / \rho_{2}$, in which $\lambda_{1}$ and $\lambda_{2}$ are positive constants. If $\rho_{1}^{2}>\lambda_{1}$ and $\rho_{2}^{2}<\lambda_{2}$, one has $0>w_{1}>-1$ and $w_{2}<-1$. This system will cross the boundary of -1 at some time because $\rho_{2}$ is always increasing and $\rho_{1}$ decreasing. The sound speed squares are $c_{s i}^{2}=-w_{i}>0$ with $i=1,2$, hence the system will be free of the difficulties associated with the singularity and the classical instability which exist in the model of a single fluid. Furthermore, the final state of this system will be characterized by $w=-1$, the universe will approach the de Sitter space in the far future. In such a scenario there will be no big rip. In the framework of the field theory, the simple way to introduce the extra degree of freedom for the quintom model is the double scalar fields model with one being quintessence-like and one phantom-like. We should point out that when adding extra degrees of freedom in the above way, this does not help solve the cosmological constant problem and nor can it help solve the coincidence problem, since for the component where $w<-1$ it cannot have the property of tracking behavior and has to be fine tuned ${ }^{1}$. The above way of introducing more components provides the simplest possibility of quintom model building.

There is another possibility of introducing the extra degrees of freedom for the realization of the transition from the quintessence phase to the phantom phase. This is the model proposed in Ref. [41] by introducing higher derivative operators to the Lagrangian. Specifically in [41] we considered a model with the Lagrangian

$$
\mathcal{L}=-\frac{1}{2} \nabla_{\mu} \phi \nabla^{\mu} \phi+\frac{c}{2 M^{2}} \square \phi \square \phi-V(\phi)
$$

where $\square \equiv \nabla_{\mu} \nabla^{\mu}$ is the d'Alembertian operator. The term related to the d'Alembertian operator is absent in the quintessence, phantom and the k-essence model, which is the key to make the model possible for $w$ to cross over -1 . We have proven in [4] this Lagrangian is equivalent to an effective two-field model

$$
\mathcal{L}=-\frac{1}{2} \nabla_{\mu} \psi \nabla^{\mu} \psi+\frac{1}{2} \nabla_{\mu} \chi \nabla^{\mu} \chi-V(\psi-\chi)-\frac{M^{2}}{2 c} \chi^{2},
$$

with the following definition

$$
\begin{aligned}
\chi & =\frac{c}{M^{2}} \square \phi, \\
\psi & =\phi+\chi .
\end{aligned}
$$

Note that the redefined fields $\psi$ and $\chi$ have opposite signs in their kinetic terms. One might be able to derive the higher derivative terms in the effective Lagrangian (18) from fundamental theories. In fact it has been shown in the

\footnotetext{
${ }^{1}$ For the k-essence field where $w<-1$, it also needs to be fine tuned.
} 
literature that this type of operators does appear as some quantum corrections or due to the non-local physics in the string theory [60, 61, 62]. With the higher derivative terms to the Einstein gravity, the theory is shown to become renormalizable 63. which has attracted many attentions. In fact the canonical form for the higher derivative theory has been put forward by Ostrogradski about one and a half century ago [64]. In short, it is interesting and worthwhile to study further the implications of models with higher derivatives in cosmology (for a recent study see e.g. [52]).

\section{PERTURBATIONS OF THE QUINTOM MODEL}

The quintom scenario as we have argued above needs extra degrees of freedom to the conventional models of a single scalar field, such as quintessence, phantom and k-essence and the simple realization of the quintom is a model with two scalar fields or "equivalently" two scalar fields for the case with the higher derivative operators. In the discussions below on the perturbations we will restrict ourselves to the two-field model of quintom with the following lagrangian:

$$
\mathcal{L}=\mathcal{L}_{Q}+\mathcal{L}_{P}
$$

where

$$
\mathcal{L}_{Q}=\frac{1}{2} \partial_{\mu} \phi_{1} \partial^{\mu} \phi_{1}-V_{1}\left(\phi_{1}\right)
$$

describes the quintessence component, and

$$
\mathcal{L}_{P}=-\frac{1}{2} \partial_{\mu} \phi_{2} \partial^{\mu} \phi_{2}-V_{2}\left(\phi_{2}\right)
$$

for the phantom component. The background equations of motion for the two scalar fields $\phi_{i}(i=1,2)$ are

$$
\ddot{\phi_{i}}+2 \mathcal{H} \dot{\phi}_{i} \pm a^{2} \frac{\partial V_{i}}{\partial \phi_{i}}=0
$$

where the positive sign is for the quintessence and the minus sign for the phantom. In general there will be couplings between the two scalar fields, here for simplicity we neglect them.

For a complete study on the perturbations, the fluctuations of the fields as well as those of the metric need to be considered. In the conformal Newtonian gauge the perturbed metric is given by

$$
d s^{2}=a^{2}(\tau)\left[(1+2 \Psi) d \tau^{2}-(1-2 \Phi) d x^{i} d x_{i}\right],
$$

Using the notations of [65], the perturbation equations satisfied by each of the components of the quintom model (22) are:

$$
\begin{aligned}
& \dot{\delta}_{i}=-\left(1+w_{i}\right)\left(\theta_{i}-3 \dot{\Phi}\right)-3 \mathcal{H}\left(\frac{\delta P_{i}}{\delta \rho_{i}}-w_{i}\right) \delta_{i}, \\
& \dot{\theta}_{i}=-\mathcal{H}\left(1-3 w_{i}\right) \theta_{i}-\frac{\dot{w}_{i}}{1+w_{i}} \theta_{i}+k^{2}\left(\frac{\delta P_{i} / \delta \rho_{i}}{1+w_{i}} \delta_{i}-\sigma_{i}+\Psi\right),
\end{aligned}
$$

where

$$
\begin{gathered}
\theta_{i}=\left(k^{2} / \dot{\phi}_{i}\right) \delta \phi_{i}, \quad \sigma_{i}=0 \\
w_{i}=\frac{P_{i}}{\rho_{i}}
\end{gathered}
$$

and

$$
\delta P_{i}=\delta \rho_{i}-2 V_{i}^{\prime} \delta \phi_{i}=\delta \rho_{i}+\frac{\rho_{i} \theta_{i}}{k^{2}}\left[3 \mathcal{H}\left(1-w_{i}^{2}\right)+\dot{w}_{i}\right] .
$$

Combining Eqs. (27), 281) and (31), we have

$$
\begin{aligned}
\dot{\delta}_{i} & =-\left(1+w_{i}\right)\left(\theta_{i}-3 \dot{\Phi}\right)-3 \mathcal{H}\left(1-w_{i}\right) \delta_{i}-3 \mathcal{H} \frac{\dot{w}_{i}+3 \mathcal{H}\left(1-w_{i}^{2}\right)}{k^{2}} \theta_{i}, \\
\dot{\theta}_{i} & =2 \mathcal{H} \theta_{i}+\frac{k^{2}}{1+w_{i}} \delta_{i}+k^{2} \Psi .
\end{aligned}
$$


Since the quintom model in (22) is essentially a combination of a quintessence and a phantom field, one obtains the perturbation equations of quintom by combining the equations above together. The corresponding variables for the quintom system are

$$
\begin{gathered}
w_{\text {quintom }}=\frac{\sum_{i} P_{i}}{\sum_{i} \rho_{i}}, \\
\delta_{\text {quintom }}=\frac{\sum_{i} \rho_{i} \delta_{i}}{\sum_{i} \rho_{i}},
\end{gathered}
$$

and

$$
\theta_{\text {quintom }}=\frac{\sum_{i}\left(\rho_{i}+p_{i}\right) \theta_{i}}{\sum_{i}\left(\rho_{i}+P_{i}\right)}
$$

Note that for the quintessence component, $-1 \leq w_{1} \leq 1$, while for the phantom component, $w_{2} \leq-1$.

With the two fields the quintom model in (22) will be characterized by the potential $V_{i}$ in (23) and (24). In this paper we take $V_{i}\left(\phi_{i}\right)=\frac{1}{2} m_{i}^{2} \phi_{i}^{2}$. In general the perturbations of $\phi_{i}$ today stem from two origins, the adiabatic and the isocurvature modes. If we use the gauge invariant variable $\zeta_{i}=-\Phi-\mathcal{H} \frac{\delta \rho_{i}}{\rho_{i}}$ instead of $\delta_{i}$, and the relation $\Phi=\Psi$ in the universe without anisotropic stress, the equations (32) and (33) can be rewritten as,

$$
\begin{aligned}
& \dot{\zeta}_{i}=-\frac{\theta_{i}}{3}-C_{i}\left(\zeta_{i}+\Phi+\frac{\mathcal{H}}{k^{2}} \theta_{i}\right) \\
& \dot{\theta}_{i}=2 \mathcal{H} \theta_{i}+k^{2}\left(3 \zeta_{i}+4 \Phi\right)
\end{aligned}
$$

where

$$
C_{i}=\frac{\dot{w}_{i}}{1+w_{i}}+3 \mathcal{H}\left(1-w_{i}\right)=\partial_{0}\left[\ln \left(a^{6}\left|\rho_{i}+p_{i}\right|\right)\right] .
$$

$\zeta_{\alpha}$ is the curvature perturbation on the uniform-density hypersurfaces for the $\alpha$-component in the universe [66]. Usually, the isocurvature perturbations of $\phi_{i}$ are characterized by the differences between the curvature perturbation of the uniform- $\phi_{i}$-density hypersurfaces and that of the uniform-radiation-density hypersurfaces,

$$
S_{i r} \equiv 3\left(\zeta_{i}-\zeta_{r}\right),
$$

where the subscript $r$ represents radiation. In this paper, we assume there is no matter isocurvature perturbations, so $\zeta_{m}=\zeta_{r}$. Eliminating $\zeta_{i}$ in equations (37) and (38), we obtain a second order equation for $\theta_{i}$,

$$
\ddot{\theta}_{i}+\left(C_{i}-2 \mathcal{H}\right) \dot{\theta}_{i}+\left(C_{i} \mathcal{H}-2 \dot{\mathcal{H}}+k^{2}\right) \theta_{i}=k^{2}\left(4 \dot{\Phi}+C_{i} \Phi\right) .
$$

This is an inhomogeneous differential equation, the general solution to it is the sum of a general solution to its homogeneous part and a special integration. In the following, we will show that the special integration corresponds to the adiabatic perturbation. Before the era of dark energy domination, the universe is dominated by some background fluids, for instance, the radiation or the matter. The perturbation equations of the background fluid are,

$$
\begin{aligned}
& \dot{\zeta}_{f}=-\theta_{f} / 3, \\
& \dot{\theta}_{f}=-\mathcal{H}\left(1-3 w_{f}\right) \theta_{f}+k^{2}\left[3 w_{f} \zeta_{f}+\left(1+3 w_{f}\right) \Phi\right] .
\end{aligned}
$$

From the Poisson equation

$$
-\frac{k^{2}}{\mathcal{H}^{2}} \Phi=\frac{9}{2} \sum_{\alpha} \Omega_{\alpha}\left(1+w_{\alpha}\right)\left(\zeta_{\alpha}+\Phi+\frac{\mathcal{H}}{k^{2}} \theta_{\alpha}\right) \simeq \frac{9}{2}\left(1+w_{f}\right)\left(\zeta_{f}+\Phi+\frac{\mathcal{H}}{k^{2}} \theta_{f}\right)
$$

we have approximately on large scales,

$$
\Phi \simeq-\zeta_{f}-\frac{\mathcal{H}}{k^{2}} \theta_{f}
$$


Combining these equations above with $\mathcal{H}=2 /\left[\left(1+3 w_{f}\right) \tau\right]$, we get (note numerically $\theta_{f} \sim \mathcal{O}\left(k^{2}\right) \zeta_{f}$ )

$$
\begin{aligned}
& \zeta_{f}=-\frac{5+3 w_{f}}{3\left(1+w_{f}\right)} \Phi=\text { Const. }, \\
& \theta_{f}=\frac{k^{2}\left(1+3 w_{f}\right)}{3\left(1+w_{f}\right)} \Phi \tau .
\end{aligned}
$$

So, we can see from Eq. (41) that there is a special solution to it which is given approximately on large scales by ,

$$
\theta_{i}^{a d}=\theta_{f}
$$

and from Eq. (38) we have,

$$
\zeta_{i}^{a d}=\zeta_{f} .
$$

This shows that the special integration to Eq. (41) has the meaning that it corresponds to the adiabatic perturbation. Hence, for the sake of isocurvature perturbations of $\phi_{i}$, we can only consider the solution to the homogeneous part of Eq. (41),

$$
\ddot{\theta}_{i}+\left(C_{i}-2 \mathcal{H}\right) \dot{\theta}_{i}+\left(C_{i} \mathcal{H}-2 \dot{\mathcal{H}}+k^{2}\right) \theta_{i}=0 .
$$

These solutions are represented by $\theta_{i}^{i s o}$ and $\zeta_{i}^{i s o}$. The relation between them is

$$
\zeta_{i}^{i s o}=\frac{\dot{\theta}_{i}^{i s o}-2 \mathcal{H} \theta_{i}^{i s o}}{3 k^{2}} .
$$

Since the general solution of $\zeta_{i}$ is

$$
\zeta_{i}=\zeta_{i}^{a d}+\zeta_{i}^{i s o}=\zeta_{r}+\zeta_{i}^{i s o},
$$

the isocurvature perturbations are simply $S_{i r}=3 \zeta_{i}^{i s o}$.

In order to solve Eq. (48), we need to know the forms of $C_{i}$ and $\mathcal{H}$ as functions of time $\tau$. For this purpose, we solve the background equations (25). In radiation dominated period, $a=A \tau, \mathcal{H}=1 / \tau$ and we have

$$
\phi_{1}=\tau^{-1 / 2}\left[A_{1} J_{1 / 4}\left(\frac{A}{2} m_{1} \tau^{2}\right)+A_{2} J_{-1 / 4}\left(\frac{A}{2} m_{1} \tau^{2}\right)\right],
$$

and

$$
\phi_{2}=\tau^{-1 / 2}\left[\tilde{A}_{1} I_{1 / 4}\left(\frac{A}{2} m_{2} \tau^{2}\right)+\tilde{A}_{2} I_{-1 / 4}\left(\frac{A}{2} m_{2} \tau^{2}\right)\right]
$$

respectively, where $A, A_{i}$ and $\tilde{A}_{i}$ are constants, $J_{\nu}(x)$ is the $\nu$ th order of Bessel function and $I_{\nu}(x)$ is the $\nu$ th order of modified Bessel function. Usually the masses are small in comparison with the expansion rate in the early universe $m_{i} \ll \mathcal{H} / a$, we can approximate the (modified) Bessel functions as $J_{\nu}(x) \sim x^{\nu}\left(c_{1}+c_{2} x^{2}\right)$ and $I_{\nu}(x) \sim x^{\nu}\left(\tilde{c}_{1}+\tilde{c}_{2} x^{2}\right)$. We note that $J_{-1 / 4}$ and $I_{-1 / 4}$ are divergent when $x \rightarrow 0$. Given these arguments one can see that this requires large initial values of $\phi_{i}$ and $\dot{\phi}_{i}$ if $A_{2}$ and $\tilde{A}_{2}$ are not vanished. If we choose small initial values, which is the natural choice if the dark energy fields are assumed to survive after inflation, only $A_{1}$ and $\tilde{A}_{1}$ modes exist, so $\dot{\phi}_{i}$ will be proportional to $\tau^{3}$ in the leading order. Thus, the parameters $C_{i}$ in equation (39) will be $C_{i}=10 / \tau$ (we have used $\left|\rho_{i}+p_{i}\right|=\dot{\phi}_{i}^{2} / a^{2}$ ). So, we get the solution to Eq. [48),

$$
\theta_{i}^{i s o}=\tau^{-4}\left[D_{i 1} \cos (k \tau)+D_{i 2} \sin (k \tau)\right] .
$$

$\theta_{i}^{i s o}$ oscillates with an amplitude damping with the expansion of the universe. The isocurvature perturbations $\zeta_{i}^{\text {iso }}$ decrease rapidly. If we choose large initial values for $\phi_{i}$ and $\dot{\phi}_{i}, A_{2}$ and $\tilde{A}_{2}$ modes are present, $\dot{\phi}_{i}$ will be proportional to $\tau^{-2}$ in the leading order and $C_{i}=0$. Now the solution to Eq. [48) is

$$
\theta_{i}^{i s o}=\tau\left[D_{i 1} \cos (k \tau)+D_{i 2} \sin (k \tau)\right] .
$$

$\theta_{i}^{\text {iso }}$ will oscillate with a increasing amplitude, so $\zeta_{i}^{i s o}$ remains constant on large scales. 
Similarly, in matter dominated era, $a=B \tau^{2}, \mathcal{H}=2 / \tau$, the solutions for the fields $\phi_{i}$ are

$$
\phi_{1}=\tau^{-3}\left[B_{1} \sin \left(\frac{B}{3} m_{1} \tau^{3}\right)+B_{2} \cos \left(\frac{B}{3} m_{1} \tau^{3}\right)\right],
$$

and

$$
\phi_{2}=\tau^{-3}\left[\tilde{B}_{1} \sinh \left(\frac{B}{3} m_{2} \tau^{3}\right)+\tilde{B}_{2} \cosh \left(\frac{B}{3} m_{2} \tau^{3}\right)\right],
$$

respectively. We get the same conclusions as those reached by the above analysis for the radiation dominated era. If we choose small initial values at the beginning of the matter domination, we will get the isocurvature perturbations in $\phi_{i}$ decrease with time. On the contrary for large initial values, the isocurvature perturbations remain constant on large scales. This conclusion is expectable. In the case of large initial velocity, the energy density in the scalar field is dominated by the kinetic term and it behaves like the fluid with $w=1$. The isocurvature perturbation in such a fluid remains constant on large scales. In the opposite case, however, the energy density in the scalar field will be dominated by the potential energy due to the slow rolling. It behaves like a cosmological constant, and there is only tiny isocurvature perturbation in it.

We have seen that the isocurvature perturbations in quintessence-like or phantom-like field with quadratical potential decrease or remain constant on large scales depending on the initial velocities. In this sense the isocurvature perturbations are stable on large scales. The amplitude of these perturbations will be proportional to the value of Hubble rate evaluated during the period of inflation $H_{\text {inf }}$ if their quantum origins are from inflation. For a large $H_{\text {inf }}$ isocurvature dark energy perturbations may be non-negligible and will contribute to the observed CMB anisotropy [67, 68]. In the cases discussed here, however, these isocurvature perturbations are negligible. Firstly, large initial velocities are not possible if these fields survive after inflation as mentioned above. Secondly, even though the initial velocities are large at the beginning of the radiation domination, these velocities will be reduced to a small value due to the small masses and the damping effect of Hubble expansion. In general the contributions of dark energy isocurvature perturbations are not very large [69] and here for simplicity we assume $H_{\text {inf }}$ is small enough that the isocurvature contributions are negligible ${ }^{2}$. Thus we will concentrate on in next sections the effects of the adiabatic perturbations of the quintom model with two scalars considered in this paper.

\section{SIGNATURES OF QUINTOM AND THE EFFECTS OF PERTURBATIONS ON OBSERVATIONS}

Based on the perturbation equations (35) and (36), we modify the code of CAMB [70] and will study preliminarily in this chapter the observational signatures of quintom. Throughout this paper we assume a flat universe. In showing the illustrative effects for quintom we have assumed the fiducial background parameters to be $\Omega_{b}=0.042, \Omega_{c}=$ $0.231, \Omega_{D E}=0.727$, where $D E$ denotes dark energy and today's Hubble constant is fixed at $H_{0}=69.255 \mathrm{~km} / \mathrm{s}$ $\mathrm{Mpc}^{-2}$. We will calculate the effects of perturbed quintom on CMB and LSS.

In the quintom model we focus on there are two parameters: one is the quintessence mass and the other being the phantom mass. When the mass of quintessence is heavier than Hubble parameter the field will start to oscillate and consequently one will get an oscillating quintom. In the numerical discussions we will fix the mass of the phantom field to be $m_{P} \sim 2.0 \times 10^{-60} m_{p l}$. We vary the quintessence mass with the typical values being $m_{Q}=10^{-60} m_{p l}$ and $4 \times 10^{-60} m_{p l}$ respectively. We plot in Fig. 1 the equations of state as function of the scale factor for the above two sets of the parameters and their corresponding effects on the observations. One can see the obvious oscillating feature of quintom as the mass of quintessence component goes heavier. After touching the $w=-1$ pivot for several times, $w$ crosses -1 consequently where the phantom part dominates dark energy. The quintom field modifies the metric perturbations: $\delta g_{00}=2 a^{2} \Psi, \delta g_{i i}=2 a^{2} \Phi \delta_{i j}$ and consequently contribute to the late time Integrated SachsWolfe (ISW) effect. The ISW effect is an integrant of $\dot{\Phi}+\dot{\Psi}$ over conformal time and wavenumber k. The above

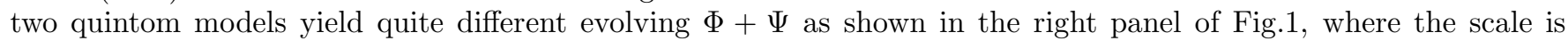
$k \sim 10^{-3} \mathrm{Mpc}^{-1}$. We can see the late time ISW effects differ significantly when dark energy perturbations are taken into account(solid lines) or not(dashed lines).

ISW effects take an important part on large angular scales of CMB and on the matter power spectrum of LSS. For a constant EOS of phantom Ref. [71] has shown that the low multipoles of CMB will get significantly enhanced when

\footnotetext{
${ }^{2}$ We assume in the next section when the mass of quintessence is larger by an order and oscillates during late time evolutions, the adiabatic condition still satisfies well.
} 
dark energy perturbations are neglected. On the other hand for a matter like scalar field where the equation of state is around zero, perturbations will also play an important role on the large scales of CMB, as shown in ref. [13]. Our results on CMB and LSS reflect the two combined effects of phantom and oscillating quintessence. Note that in the early studies of quintessence effects on CMB, one usually considers a constant $w_{\text {eff }}$ instead:

$$
w_{e f f} \equiv \frac{\int d a \Omega(a) w(a)}{\int d a \Omega(a)}
$$

however this is not enough for the study of effects on SN, nor for CMB when the EOS of dark energy has a very large variation with redshift, such as the model of oscillating quintom considered in this paper.

To face the oscillating model of quintom with the current observations, we make a preliminary fitting to the first year Wilkinson Microwave Anisotropy Probe(WMAP) TT and the TE temperature-polarization cross-power spectrum as well as the recently released 157 "Gold" SN data 22]. Following Refs. 72, 73] in all the fittings below we will fix $\tau=0.17, \Omega_{m} h^{2}=0.135$ and $\Omega_{b} h^{2}=0.022$, we set the spectral index as $n_{S}=0.95$ and the amplitude of the primordial spectrum will be used as a continuous parameter. In the fittings of oscillating quintom we've fixed the mass of phantom to be $m_{P} \sim 6.2 \times 10^{-61} m_{p l}$. Fig 2] delineates $3 \sigma$ WMAP and SN constraints on the two-field quintom model, it also shows the corresponding best fit values. In the labels $m_{Q}$ and $m_{P}$ stand for the mass of quintessence and phantom respectively. The left panel of Fig 2 shows the separate WMAP and SN constraints. The green(shaded) area is WMAP constraints on models where dark energy perturbations have been included and the blue area(contour with solid lines) is without dark energy perturbations. The perturbations of dark energy have no effects on the geometric constraint of SN. The right panel shows the combined WMAP and SN constraints on the two-field quintom model with perturbations (green/shaded region) and without perturbations (red region/contour with solid lines). We find the confidence regions do show a large difference when the perturbations of dark energy have been taken into account or not.

So far we have investigated the imprints of oscillating quintom on CMB and LSS. Now we consider another example where $w$ crosses -1 smoothly without oscillation. It is interesting to study the effects of this type of quintom model with its effective equation of state defined in (57) exactly equal to -1 on CMB and matter power spectrum. This study will help to distinguish the quintom model from the cosmological constant. We have realized such a model of quintom in the lower right panel of Fig [3] which can be easily given in the two-field model with lighter quintessence mass. In this example we have set $m_{Q} \sim 2.6 \times 10^{-61} m_{p l}, m_{P} \sim 6.2 \times 10^{-61} m_{p l}$. We assume there are no initial kinetic energy. The initial values of the quintessence component is set as $\phi_{1 i}=0.226 m_{p l}$ and the phantom part: $\phi_{2 i}=6.64 \times 10^{-3} m_{p l}$. We find the EOS of quintom crosses -1 at $z \sim 0.15$, which is consistent with the latest SN results.

The model of quintom, which is mainly favored by current SN only, needs to be confronted with other observations in the framework of concordance cosmology. SN making the only direct detection of dark energy, this model is most promising to be distinguished from the cosmological constant and other dynamical dark energy models which do not get across -1 by future SN projects on the low redshift(for illustrations see e.g. [32]). This is also the case for the model of quintom in the full parameter space: it can be most directly tested in low redshift Type Ia supernova surveys. In the upper left panel of Fig 3 we delineate the different ISW effects among the cosmological constant (red/light solid), the quintom model which gives $w_{e f f}=-1$ with (blue/dark solid) and without(blue dashed) perturbations. Similar to the previous oscillating case, the difference is very large when switching off quintom perturbations and much smaller when including the perturbations. In the upper right panel we find the quintom model cannot be distinguished from a cosmological constant in light of WMAP. The two models almost give exactly the same results in CMB TT and TE power spectra when including the perturbations. We find the difference in CMB is hardly distinguishable even by cosmic variance.

Given the fact aobve that from CMB observations quintom with $w_{\text {eff }}=-1$ makes no distinctive signatures, now we discuss briefly the signatures in some other observations. To do that we need to consider the physical observables which can be affected by the evolving $w$ sensitively. In comparison with the cosmological constant such a quintom model gives a different evolution of expansion history of universe, such as altering the epoch of matter-radiation equality. The Hubble expansion rate $H$ is :

$$
H \equiv \frac{\dot{a}}{a^{2}}=H_{0}\left[\Omega_{m} a^{-3}+\Omega_{r} a^{-4}+X\right]^{1 / 2}
$$

where $\mathrm{X}$, the energy density ratio of dark energy between the early epochs and today, is quite different for the quintom-CDM and $\Lambda \mathrm{CDM}$. In the $\Lambda \mathrm{CDM}$ scenario, $\mathrm{X}$ is simply a constant while in general for dark energy models with varying energy density or EOS,

$$
X=\Omega_{D E} a^{-3} e^{-3 \int w(a) d \ln a} .
$$


The two models will give different Hubble expansion rates. This is also the case between the quintom model with $w_{e f f}=-1$ in the left panel of Fig [3] and a cosmological constant. Different $H$ leads directly to different behaviors of the growth factor. In the linear perturbation theory all Fourier modes of the matter density perturbations grow at the same rate. The matter density perturbations are independent of $k$ :

$$
\ddot{\delta}_{k}+\mathcal{H} \dot{\delta}_{k}-4 \pi G a^{2} \rho_{\mathrm{M}} \delta_{k}=0
$$

The growth factor $D_{1}(a)$ characterizes the growth of the matter density perturbations: $D_{1}(a)=\delta_{k}(a) / \delta_{k}(a=1)$ and is normalized to unity today. In the matter-dominated epoch we have $D_{1}(a)=a$. Analytically $D_{1}(a)$ is often approximated by the Meszaros equation [75]:

$$
D_{1}(a)=\frac{5 \Omega_{m} H(a)}{2 H_{0}} \int_{0}^{a} \frac{d a^{\prime}}{\left(a^{\prime} H\left(a^{\prime}\right) / H_{0}\right)^{3}},
$$

where we can easily see the difference between the model of quintom and a cosmological constant due to the different Hubble expansion rates. More strictly one needs to solve Eq.(60) numerically. In the lower left panel of Fig [3 we show the difference of $D_{1}(a)$ between the quintom with $w_{\text {eff }}=-1$ and the cosmological constant. The difference in the linear growth function is considerably large in the late time evolution and possibly distinguishable in future LSS surveys and in weak gravitational lensing (WGL) observations. WGL has emerged with a direct mapping of cosmic structures and it has been recently shown that the method of cosmic magnification tomography can be extremely efficient [76], which leaves a promising future for breaking the degeneracy between quintom and a cosmological constant.

\section{PERTURBATIONS OF PARAMETRIZED QUINTOM AND THE EFFECTS ON THE OBSERVATIONS}

There have been many studies in the literature in the fittings of the dark energy with parametrized EOS, such as the linear parametrization $w=w_{0}+w_{1} z[77]$ to SN and other observations such as CMB and LSS. For the latter observations the perturbations of dark energy need to be considered. However, at the point of $w=-1$, as pointed out in Section II one would be encountered with the singularity of the isentropic sound speed. Moreover in the perturbation equation (28) one will get infinite $\dot{\theta}$. For the physical quantity $(\rho+P) \theta$ in the model of the single field of quintessence, it is not divergent at $w=-1$, i.e. $\theta \rightarrow \infty$ but $(\rho+P) \theta=k^{2} \dot{\phi} \delta \phi=0$, however for the model with parametrized EOS one will generically have an unphysical divergence when $\dot{w} \neq 0$ at the cosmological constant boundary. The detailed explanation is given as follows: firstly from Eq.(28) one will get infinite $\dot{\theta}$ and the physical continuity implies that one will also get $\theta \rightarrow \infty$ at $w=-1$. Introducing the new physical quantity which is relevant to the CMB observations:

$$
\mathcal{V} \equiv(1+w) \theta
$$

Eqs.(32, 331) can be rewritten now as:

$$
\begin{aligned}
\dot{\delta} & =-\mathcal{V}+(1+w) 3 \dot{\Phi}-3 \mathcal{H}(1-w) \delta-3 \mathcal{H} \frac{\dot{w} /(1+w)+3 \mathcal{H}(1-w)}{k^{2}} \mathcal{V}, \\
\dot{\mathcal{V}} & =2 \mathcal{H} \mathcal{V}+k^{2} \delta+\frac{\dot{w}}{1+w} \mathcal{V}+k^{2}(1+w) \Psi
\end{aligned}
$$

We can easily see that $\dot{\mathcal{V}} \rightarrow \infty$ when $\dot{w} \neq 0$ at $w=-1$.

We should point out that both the scalar fields and fluids obey the same form of equations on the evolution of perturbations: Eqs.(2728), and the only difference comes from the term of $\delta P_{i} / \delta \rho_{i}$. If one starts from Eqs. (32) 331) and study the effects of dark energy by parametrizing the EOS, this is equivalent to the description of the effects of the scalar field and is identical to work starting with dark energy potentials. If in models with the parametrized EOS we have $w$ always in the range $[-1,1]$, or $w \leq-1$ for $0<a<\infty$, there will be no unphysical divergence and this equivalently describes the single field of quintessence or phantom 78$]$. For example in model with $w=w_{0}+w_{1} \sin (\ln a)$, if we restrict $w_{0}=0$ and $\left|w_{1}\right| \leq 1$ then Eqs. (63, 64) will always be continuous. However when the parameter space is enlarged to include $\dot{w} \neq 0$ at $w=-1$ Eqs. 663 64) will be unphysical.

We emphasize that the above discussions are valid only for models with a single field. For models with multi fields we have shown explicitly in the previous sections the perturbation equations Eqs. (32) 331) are continuous during the crossing of the cosmological constant boundary. It is similar for models with two fluids or models with two components of parametrized EOS: $w=\Sigma \Omega_{i} w_{i}$ where each component $w_{i}$ does not evolve across -1 . This implies, however, in the 
fitting of the models to the observational data the parameters introduced for the EOS should be doubled if allowing the $\operatorname{EOS} w$ to vary and get across -1 . Certainly this is not practically applicable. It would be nice to develop a technique to include the perturbations which approximates well to the quintom, meanwhile not introducing the extra degrees of freedom to the models considered widely in the literature with parametrized EOS. We will make a proposal for it below.

First of all we consider a system of quintom with two fields as above, $\phi_{1}$ being quintessence-like and $\phi_{2}$ being phantom-like, but restrict the EOS of the system not to cross over -1 . In this case we will show the background of this system is equivalent to a model with an effective single scalar field denoted by $\chi$. By definition the pressure $\mathrm{P}$ and energy density $\rho$ of the $\chi$ field should be equal to the two-field case. When the kinetic term of $\phi_{1}$ is larger than that of the phantom part $\phi_{2}$, the whole system of dark energy gives rise to an EOS larger than -1 and the effective $\chi$ behaves like a quintessence. On the contrary when ${\dot{\phi_{1}}}^{2}-{\dot{\phi_{2}}}^{2} \leq 0, \chi$ is a phantom field. Hence the kinetic and potential terms of $\chi$, in terms of $\phi_{1}$ and $\phi_{2}$, can be expressed as

$$
\pm \dot{\chi}^{2}=\dot{\phi}_{1}^{2}-\dot{\phi}_{2}^{2}
$$

and

$$
V(\chi)=V\left(\phi_{1}\right)+V\left(\phi_{2}\right)
$$

where the "+" sign in Eq. [65 is for the case where the total EOS of dark energy is quintessence-like and the "-" sign for phantom-like evolutions. We can directly reconstruct the potential and time evolutions of $\chi$. For example if we set the potentials of the two fields to be both linear:

$$
V_{i}\left(\phi_{i}\right)=V_{0 i}+\lambda_{i} \phi_{i}
$$

in the early epochs of radiation and matter domination dark energy fields are slow rolling and

$$
\phi_{1}{ }^{\prime} \sim-\lambda_{1} / 3 H, \phi_{2}{ }^{\prime} \sim \lambda_{2} / 3 H
$$

where prime denotes derivative respects to the physical time. For the whole system in the quintessence phase $\phi_{1}$ will have a larger kinetic energy, and in the radiation dominant epoch

$$
H=1 / 2 t \quad, \quad \chi^{\prime}= \pm \frac{2}{3} t \sqrt{\lambda_{1}^{2}-\lambda_{2}^{2}}
$$

On the other hand from Eq. (66) we have

$$
V_{, \chi}(\chi) \chi^{\prime}=V_{, \phi_{1}}\left(\phi_{1}\right) \phi_{1}^{\prime}+V_{,_{2}}\left(\phi_{2}\right) \phi_{2}^{\prime},
$$

combining Eqs.686970) we can easily get

$$
V_{, \chi}(\chi)=\mp \sqrt{\lambda_{1}^{2}-\lambda_{2}^{2}},
$$

consequently the effective potential of $\chi$ analytically is

$$
V(\chi)= \pm \sqrt{\lambda_{1}^{2}-\lambda_{2}^{2}}\left(\chi-\chi_{0}\right)
$$

where $\chi_{0}$ can be easily set by the initial conditions of $\phi_{i}$ and the sign of "+" or "-" is somewhat optional. The arguments above applies for the case when the total EOS of the system is restricted to be no larger than -1 , the effective scalar will behave like phantom.

On the evolution of perturbations we can see from Eqs.2728) that the phantom and the quintessence fields obey the same equations. As shown in Section III although generically the two field model would have non-vanishing isocurvature perturbations we can choose suitable initial conditions so that the isocurvature contributions can be safely negligible. In this sense when the total EOS does not evolve across minus unity, the whole system can be equally described by an adiabatic field: both the background evolution and the adiabatic perturbations, as shown similarly in Refs. 73, 79, 80] in the inflationary universe.

We have demonstrated in the previous paragraphs the equivalence between the two-field quintom model and the single scalar field model when the EOS of the system does not cross over -1 . However if the total equation of state for the double fields does cross over -1 , this system will not be able to be described effectively by a single scalar field. To study the perturbations of the dark energy models with EOS across -1 , we introduce a small positive constant $c$ 
to divide the whole region of the allowed value of the EOS $w$ into three parts: 1) $w>-1+c$;2) $-1+c>w>-1-c$; and 3) $w<-1-c$. For 1) the EOS is always larger than -1 and for 3) $w$ is always less than -1 . For both cases the system with two fields as shown above can be described effectively by a single scalar field with a potential satisfying [1]

$$
\pm a^{2} \frac{d^{2} V}{d \chi^{2}}=-\frac{3}{2}(1-w)\left[\frac{\ddot{a}}{a}-\mathcal{H}^{2}\left(\frac{7}{2}+\frac{3}{2} w\right)\right]+\frac{1}{1+w}\left[\frac{\dot{w}^{2}}{4(1+w)}-\frac{\ddot{w}}{2}+\dot{w} \mathcal{H}(3 w+2)\right]
$$

where "+" is for the case 1) and "-" for the case 3). One can see that $\frac{d^{2} V}{d \chi^{2}}$ is divergent and there would be a discontinuity in the derivative $V^{\prime}$ at the turning point of $w=-1$, which corresponds to $c \rightarrow 0$. As an example in Fig. 4 we give the reconstructed potential of the effective $\chi$ field for an oscillating quintom. One can see $\chi$ behaves like quintessence when $w>-1$ and like phantom when $w<-1$. The reconstructed potential is well defined except in region 2) when the EOS gets across -1, where there is a sharp discontinuity on $V^{\prime}(\chi)$.

For the case 2), different from those in 1) and 3), the perturbations cannot be fully described by a single adiabatic field. However as we learn from the above, for the realistic quintom models the perturbations in the region 2) will be continuous and not divergent, i.e. $\delta$ and $\theta$ are continuous, and the derivatives of $\delta$ and $\theta$ are finite. A good approximation to the perturbation in region 2) is requiring it to match to the regions 1) and 3) at the boundary. Practically we take $\delta$ and $\theta$ to be constant matching to regions 1 ) and 3 ) at the boundary and set

$$
\dot{\delta}=0, \quad \dot{\theta}=0 .
$$

In the numerical calculation the constant $c$ is a very small number, the approximation above lies in a very close neighborhood of $w=-1$. In practice in our numerical calculations we've limited the range to be $|\Delta w=c|<10^{-5}$. Since the region 2) is extremely limited, neglecting the evolutions of perturbations as shown in (74) is quite safe and well approximated. Thus we can use Eqs. (32) 331) to study the effects of perturbations in models with parametrized EOS. We have also numerically checked the validity of Eq.(74) and found their contributions to the observed CMB and LSS power spectra are very small. The procedure of our checking is listed as follows:

1. Start with the two-field model of quintom and record $w(a)$, compute CMB and LSS spectra with perturbations.

2. Build a code in CAMB 70] to include dark energy perturbations with parametrized EOS. Include perturbations by setting Eq. (74) and treating $\delta, \theta$ as continuous.

3. Interpolate w(a) in the code with parametrized EOS, compute CMB and LSS spectra and make comparisons with the results from step 1.

With this procedure we have considered a model of oscillating quintom and found the difference is no more than $10^{-4}$, which is safely negligible.

As examples now we study the effects of perturbations for several models with parametrized EOS in light of WMAP and SN data. The first example is given by Ref. 42], where $w$ is parametrized by

$$
w(\ln a)=w_{a}+w_{0} \cos \left[w_{1} \ln \left(a / a_{c}\right)\right]
$$

with $a$ being the scale factor. This model has a nice feature of unifying the early inflation and the current accelerated expansions. In Ref. 42] the period of oscillation has been set as long as $\sim 200$ e-folds. It is interesting to study the consequences with a shorter period. Here for illustrations we fix $w_{a}=-1, w_{1}=20$ and $a_{c}=1$. In the upper panels of Fig [5 we show the illustrative fittings when with and without the perturbations. We can see the parameter space has been enlarged a lot when including the contributions of the perturbations. For a second example we parametrize $w$ as

$$
w(\ln a)=w_{a}+w_{0} a \cos \left[w_{1} a+a_{c}\right] .
$$

In the numerical calculation we've fixed $w_{a}=-1, w_{1}=50$ and $a_{c}=0$. In the lower panels of Fig $[$ we can see the effects are still very prominent both in the separate and combined constraints, although not as strong as the example in (75). For the third example we take $w$ to be non-oscillatory:

$$
w=w_{0} /(1-\ln a),
$$

where the original form was firstly proposed in Ref. [82]. We find in our case SN constraints are very weak due to the fixed background parameters, the $1 \sigma$ regions have not been affected much by the perturbations, but the $2,3 \sigma$ regions have been enlarged significantly when the perturbations are taken into account. 


\section{CONCLUSIONS}

In this paper we have studied the perturbations of the dynamical quintom model of dark energy in a self-consistent way. It is physically significant for the inclusion of quintom perturbations, both on the theoretical grounds of model buildings and on the fittings to the observations. Due to the singularities and instabilities of perturbations at the cosmological constant boundary, we have shown a new method regarding the impossibility of k-essence as a viable quintom model. In the realistic quintom model buildings one must include the perturbations. In general one needs to add extra degrees of freedom to realize the model of quintom. In the two-field model and the model with a d'Alembertian operator the isocurvature contributions may be safely negligible in the simplest case. We have considered the implications of quintom perturbations on the observations of CMB and LSS. We have shown that the parameter space is different when one includes the perturbations of dark energy or not. In trying to constrain dark energy in a model independent way we have also proposed a method to include the perturbations for models of dark energy with parametrized EOS across -1 . With some specific examples of the parametrized EOS, we show that the parameter space which characterizes the properties of the model will get enlarged in general when including the perturbations. This will lead to important consequences in the phenomenological studies on the cosmological imprints of dynamical dark energy, including the model of quintom. A thorough investigation of current constraints on the quintom model of dark energy where dark perturbations are taken into account is beyond the scope of current paper and will be presented elsewhere 83, 84].

Overall, a dynamical quintom model is favored by current SN data and not ruled out by the combined observational constraints. There are still some inconsistencies today among different observations in the precision cosmology and the concordance $\Lambda \mathrm{CDM}$ model has not yet fitted well to the observations in a high enough confidence level, in this sense we might not be adopting the Ockham's razor with a cosmological constant. When we start from a $\Lambda$ CDM model in the probe of our universe we cannot achieve more subtle physics beyond that. This is necessary to bear in mind for us to understand the nature of dark energy with the accumulation of the observational data.

Acknowledgements: We thank the anonymous referee for helpful suggestions. We thank Robert Brandenberger, Xue-Lei Chen, Zuhui Fan, Pei-Hong Gu, Hong Li, Hiranya Peiris, Yunsong Piao, Yong-Zhong Xu and Peng-Jie Zhang for helpful discussions. We acknowledge the using of CMBfast 85, 86] in our early studies and CAMB 70, 87] for all the numerical calculations. In the fitting to WMAP we've used the code developed in Ref. 88. We thank Antony Lewis for early miscellaneous help and discussions on the cosmocoffee 89]. This work is supported in part by National Natural Science Foundation of China under Grant Nos. 90303004 and 19925523 and by Ministry of Science and Technology of China under Grant No. NKBRSF G19990754. B. F. would like to thank the hospitality of the National Astronomical Observatories, Chinese Academy of Sciences where part of this work was finished and M. L. is supported by Alexander von Humboldt Foundation.

[1] A. G. Riess et al., Astron. J. 116, 1009 (1998).

[2] S. Perlmutter et al., Astrophys. J. 517, 565 (1999).

[3] S. Weinberg, Rev. Mod. Phys. 61, 1 (1989).

[4] I. Zlatev, L.-M. Wang, and P. J. Steinhardt, Phys. Rev. Lett. 82, 896 (1999).

[5] B. Ratra and P. J. E. Peebles, Phys. Rev. D 37, 3406 (1988); P. J. E. Peebles and B. Ratra, Astrophys. J. 325, L17 (1988).

[6] C. Wetterich, Nucl. Phys. B 302, 668 (1988); C. Wetterich, Astron. Astrophys. 301, 321 (1995).

[7] G. Huey, L.-M. Wang, R. Dave, R. R. Caldwell and P. J. Steinhardt, Phys. Rev. D 59, 063005 (1999).

[8] L.-M. Wang, R. R. Caldwell, J. P. Ostriker and P. J. Steinhardt Astrophys. J. 530, 17 (2000).

[9] S. M. Carroll, W. H. Press , E. L. Turner, Ann. Rev. Astron. Astrophys. 30, 499 (1992).

[10] L. M. Krauss, M. S. Turner, Gen. Rel. Grav. 27, 1137 (1995).

[11] J. P. Ostriker, P. J. Steinhardt, Nature 377, 600 (1995).

[12] K. Coble, S. Dodelson, J. A. Frieman, Phys. Rev. D 55, 1851 (1997).

[13] R. R. Caldwell, R. Dave and P. J. Steinhardt, Phys. Rev. Lett. 80, 1582 (1998).

[14] P. M. Garnavich et al., Astrophys. J. 509, 74 (1998).

[15] R. R. Caldwell, Phys. Lett. B 545, 23 (2002).

[16] S. M. Carroll, M. Hoffman and M. Trodden, Phys. Rev. D 68, 023509 (2003); J. M. Cline, S.-Y. Jeon and G. D. Moore, Phys. Rev. D 70, 043543 (2004); R. V. Buniy and S. D.H. Hsu, hep-th/0502203

[17] e. g. P. H. Frampton, Phys. Lett. B 555, 139 (2003); V. Sahni and Y. Shtanov, J. Cosmol. Astropart. Phys. 0311, 014 (2003); B. McInnes, J. High Energy Phys. 0208, 029 (2002); V.K. Onemli and R.P. Woodard, Class. Quant. Grav. 19, 4607 (2002); V. K. Onemli and R. P. Woodard, Phys. Rev. D 70,107301 (2004); I. Y. Aref'eva, A.S. Koshelev and S.Y. Vernov, astro-ph/0412619. I. Y. Aref'eva and L. V. Joukovskaya, hep-th/0504200

[18] C. L. Bennett et al., Astrophys. J. Suppl.148, 1 (2003).

[19] J. L. Tonry et al., Astrophys. J. 594, 1 (2003). 
[20] R. A. Knop et al., Astrophys. J. 598, 102 (2003).

[21] M. Tegmark et al., Astrophys. J. 606, 702 (2004).

[22] A. G. Riess et al., Astrophys. J. 607, 665 (2004).

[23] S. W. Allen, R. W. Schmidt, H. Ebeling, A. C. Fabian, and L. van Speybroeck, Mon. Not. Roy. Astron. Soc. 353, 457 (2004).

[24] e. g. Y. Wang and M. Tegmark, Phys. Rev. Lett. 92 (2004) 241302.

[25] T. D. Saini, S. Raychaudhury, V. Sahni, and A. A. Starobinsky, Phys. Rev. Lett. 85, 1162 (2000).

[26] D. Huterer and G. Starkman, Phys. Rev. Lett. 90, 031301 (2003).

[27] R. A. Daly and S. G. Djorgovski, Astrophys. J. 612, 652 (2004).

[28] V. Sahni and A. A. Starobinsky, Int. J. Mod. Phys. D 9, 373 (2000); L. Amendola, Phys. Rev. D 62, 043511 (2000); J. Weller and A. Albrecht, Phys. Rev. Lett. 86, 1939 (2001); Z.-H. Zhu and M.-K. Fujimoto, Astrophys. J. 581, 1 (2002); T. R. Choudhury and T. Padmanabhan, Mon. Not. Roy. Astron. Soc. 344, 823 (2003); A. Melchiorri, L. Mersini, C. J. Odman and M. Trodden, Phys. Rev. D 68, 043509 (2003); E. V. Linder and A. Jenkins, Mon. Not. Roy. Astron. Soc. 346, 573 (2003); Y. Zhang, Chin. Phys. Lett. 20, 1899 (2003); T. R. Choudhury and T. Padmanabhan, Astron. Astrophys. 429, 807 (2005); D. Puetzfeld and X.-L. Chen, Class. Quant. Grav. 21, 2703 (2004); T. Deep Saini, J. Weller and S. L. Bridle, Mon. Not. Roy. Astron. Soc. 348, 603 (2004); P. Mukherjee, A. J. Banday, A. Riazuelo, K. M. Grski and B. Ratra, Astrophys. J. 598, 767 (2003); Y. Zhang, Chin. Phys. Lett. 21, 1183 (2004); F. S. N. Lobo,Phys.Rev. D 71, 084011 (2005); Phys. Rev. D 71, 124022(2005); M. Sami, A. Toporensky, P. V. Tretjakov and S. Tsujikawa, Phys. Lett. B 619, 193 (2005).

[29] M. Kunz, P.-S. Corasaniti, D. Parkinson and E. J. Copeland, Phys. Rev. D 70, 041301 (2004); P. Caresia, S. Matarrese and L. Moscardini, Astrophys. J. 605, 21 (2004); Y. Wang and P. Mukherjee, Astrophys. J. 606, 654 (2004); S. Nesseris and L. Perivolaropoulos, Phys. Rev. D 70, 043531 (2004); U. Alam, V. Sahni, T. D. Saini, and A. A. Starobinsky, Mon. Not. Roy. Astron. Soc. 354, 275 (2004); Z. H. Zhu, M. K. Fujimoto, and X. T. He, Astron. Astrophys. 417, 833 (2004); Y. Gong, Class. Quant. Grav. 22, 2121 (2005); Z.-H. Zhu, Astrophys. J. 620, 7 (2005); G. Chen and B. Ratra, Astrophys. J. 612, L1 (2004); D. A. Dicus and W. W. Repko, Phys. Rev. D 70, 083527 (2004); Z. G. Dai, E.W. Liang and D. Xu, Astrophys. J. 612, L101 (2004). Y. Wang, J. M. Kratochvil, A. Linde and M. Shmakova, J. Cosmol. Astropart. Phys. 0412, 006 (2004); L. Conversi, A. Melchiorri, L. Mersini and J. Silk, Astropart. Phys. 21 443, (2004); W. Godlowski and M. Szydlowski, Gen. Rel. Grav. 36, 767 (2004); D. Rapetti, S. W. Allen and J. Weller, Mon. Not. Roy. Astron. Soc. 360, 555 (2005); J. Simon, L. Verde and R. Jimenez, Phys. Rev. D 71, 123001 (2005).

[30] C. Csaki, N. Kaloper and J. Terning, Annals Phys 317, 410 (2005); N. Dalal, D. E. Holz, X. Chen and J. A. Frieman, Astrophys. J. 585, (2003) L11; E. Majerotto, D. Sapone and L. Amendola, astro-ph/0410543 M. P. Dabrowski and T. Stachowiak, hep-th/0411199 L. Perivolaropoulos, Phys. Rev. D 71, 063503 (2005); Z.-H. Zhu, Astrophys. J. 620, 7 (2005); Y. Gong and Y.-Z. Zhang, astro-ph/0502262 S. Hannestad, Phys.Rev.D 71, 103519 (2005); M. C. Bento, O. Bertolami, N. M. C. Santos and A. A. Sen, Phys. Rev. D71, 063501 (2005); G. Olivares, F. Atrio-Barandela and D. Pavon, Phys.Rev. D 71, 063523 (2005); W. Wang, Y.-X. Gui, S.-H. Zhang, G.-H. Guo and Y. Shao, astro-ph/0504094 M. Szydlowski, W. Godlowski, A. Krawiec and J. Golbiak, astro-ph/0504464 L. Perivolaropoulos, astro-ph/0504582 X. Zhang and F.-Q. Wu, astro-ph/0506310 B. Wang, Y. Gong and E. Abdalla, hep-th/0506069 M.-X. Luo and Q.-P. Su, astro-ph/0506093 Z.-H. Zhu and J.S. Alcaniz, Astrophys. J. 620, 7 (2005); C. Csaki, N. Kaloper and J. Terning, astro-ph/0507148 M. Ishak, A. Upadhye and D. N. Spergel, astro-ph/0507184 D. Polarski and A. Ranquet, astro-ph/0507290 J. S. Alcaniz and J. A. S. Lima, astro-ph/0507372 M. Giovannini, astro-ph/0507369

[31] U. Alam, V. Sahni and A. A. Starobinsky, J. Cosmol. Astropart. Phys. 0406, 008 (2004).

[32] D. Huterer and A. Cooray, Phys. Rev. D 71, 023506 (2005).

[33] P. S. Corasaniti, M. Kunz, D. Parkinson, E. J. Copeland and B. A. Bassett, Phys. Rev. D 70, 083006 (2004);

[34] S. Hannestad and E. Mortsell, J. Cosmol. Astropart. Phys. 0409001 (2004); A. Upadhye, M. Ishak and P. J. Steinhardt, astro-ph/0411803

[35] B. Feng, X. Wang, and X. Zhang, Phys. Lett. B 607, 35, (2005).

[36] U. Seljak et al., astro-ph/0407372

[37] Ch. Yeche, A. Ealet, A. Refregier, C. Tao, A. Tilquin, J.-M. Virey and D. Yvon, astro-ph/0507170

[38] A. Vikman, Phys. Rev. D 71, 023515, (2005).

[39] C. Armendariz-Picon, V. Mukhanov and P. J. Steinhardt, Phys. Rev. Lett. 85, 4438 (2000); Phys. Rev. D 63, 103510 (2001).

[40] Z.-K. Guo, Y.-S. Piao, X. Zhang, and Y.-Z. Zhang, Phys. Lett. B 608, 177, (2005).

[41] M.-Z. Li, Bo Feng, Xin-min Zhang, hep-ph/0503268

[42] B. Feng, M. Li, Y.-S. Piao and X. Zhang, astro-ph/0407432

[43] e. g. Y.-S. Piao and E Zhou, Phys. Rev. D 68, 083515, (2003); Y.-S. Piao and Y.-Z. Zhang, Phys. Rev. D 70, 063513, (2004).

[44] Y.-H. Wei and Y. Tian, Class. Quant. Grav. 21, 5347, (2004).

[45] X. Zhang, hep-ph/0410292

[46] W. Hu, Phys.Rev. D71 (2005) 047301.

[47] J.-Q. Xia, B. Feng, and X. Zhang, astro-ph/0411501

[48] R. R. Caldwell and M. Doran, astro-ph/0501104

[49] B.-J. Li, M.-C. Chu, K.-C. Cheung, A. Tang, astro-ph/0501367

[50] X.-F. Zhang, H. Li, Y.-S. Piao, and X. Zhang, astro-ph/0501652

[51] H. Wei and R.-G. Cai, hep-th/0501160 R.-G. Cai, H.-S. Zhang and A. Wang, hep-th/0505186 A. A. Andrianov, F. Cannata and A. Y. Kamenshchik, gr-qc/0505087 X. Zhang, astro-ph/0504586 Q. Guo and R.-G. Cai, gr-qc/0504033 B. 
McInnes, Nucl. Phys. B 718, 55 (2005); E. Elizalde, S. Nojiri, S. D. Odintsov and P. Wang, Phys. Rev. D 71, 103504

(2005); I. Y. Aref'eva, A. S. Koshelev, and S. Yu. Vernov, astro-ph/0507067

[52] A. Anisimov, E. Babichev and A. Vikman, J. Cosmol. Astropart. Phys. 0506, 006 (2005).

[53] S. Nojiri, S. D. Odintsov and S. Tsujikawa, Phys. Rev. D 71, 063004 (2005).

[54] H. Stefancic, astro-ph/0504518

[55] R. Jackiw, V. P. Nair, S. Y. Pi and A. P. Polychronakos, J. Phys. A 37, R327-R432, (2004).

[56] H. Kodama and M. Sasaki, Prog. Theor. Phys. Suppl. 78 , 1 (1984).

[57] Y. Aharonov, A. Komar, and L. Susskind, Phys. Rev. 182, 1400, (1969).

[58] For early studies see e. g. J. D. Barrow, Class. Quant. Grav. 21, L79 (2004).

[59] e. g. N. Bilic, G. B. Tupper and R. D. Viollier Phys. Lett. B 535, 17 (2002).

[60] J. Z. Simon, Phys. Rev. D 41, 3720 (1990).

[61] A. Elizer and R. P. Woodard, Nucl. Phys. B 325, 389 (1989).

[62] T. G. Erler and D. J. Gross, hep-th/0406199

[63] K. S. Stelle, Phys. Rev. D 16, 953 (1977).

[64] M. Ostrogradski, Mem. Ac. St. Petersbourg VI 4, 385 (1850).

[65] C. -P. Ma and E. Berschinger, Astrophys. J. 455 7, (1995).

[66] D. Wands, K.A. Malik, D.H. Lyth, and A.R. Liddle, Phys. Rev. D 62, 043527, (2000).

[67] M. Kawasaki, T. Moroi, and T. Takahashi, Phys. Rev. D 64, 083009 (2001).

[68] T. Moroi and T. Takahashi, Phys. Rev. Lett. 92, 091301 (2004).

[69] For a recent study see C. Gordon and D. Wands, Phys.Rev. D 71, 123505 (2005).

[70] A. Lewis, A. Challinor and A. Lasenby, Astrophys. J. 538, 473 (2000).

[71] J. Weller and A. M. Lewis, Mon. Not. Roy. Astron. Soc. 346, 987 (2003).

[72] C. R. Contaldi, M. Peloso, L. Kofman and A. Linde, J. Cosmol. Astropart. Phys. 0307, 002 (2003).

[73] B. Feng and X. Zhang, Phys. Lett. B 570, 145 (2003).

[74] A. Kogut et al., Astrophys. J. Suppl.148, 161 (2003); G. Hinshaw et al., Astrophys. J. Suppl.148, 135 (2003).

[75] See e.g. S. Dodelson, Modern Cosmology, Academic Press, 2003.

[76] B. Jain and A. Taylor, Phys. Rev. Lett. 91, 141302 (2003); P. Zhang and U.-L. Pen, astro-ph/0504551 P. Zhang and U.-L. Pen, astro-ph/0506740

[77] A. R. Cooray and D. Huterer, Astrophys. J. 513, L95 (1999).

[78] e.g. Z.-K. Guo, N. Ohta and Y.-Z. Zhang, astro-ph/0505253

[79] C. Gordon, D. Wands, B. A. Bassett and R. Maartens, Phys. Rev. D 63, 023506 (2001).

[80] X.-J. Bi, B. Feng and X.-M. Zhang, hep-ph/0309195

[81] R. Dave, R. R. Caldwell and P. J. Steinhardt, Phys.Rev. D 66, 023516 (2002).

[82] C. Wetterich, Phys. Lett. B 594, 17 (2004).

[83] B. Feng et al., to appear.

[84] X. Zhang, hep-ph/0510072, see Figures 3-4 of the paper.

[85] U. Seljak and M. Zaldarriaga, Astrophys. J. 469, 437 (1996).

[86] http://cmbfast.org/

[87] http://camb.info/

[88] L. Verde et al., Astrophys. J. Suppl. 148, 195 (2003).

[89] http://cosmocoffee.info/ 

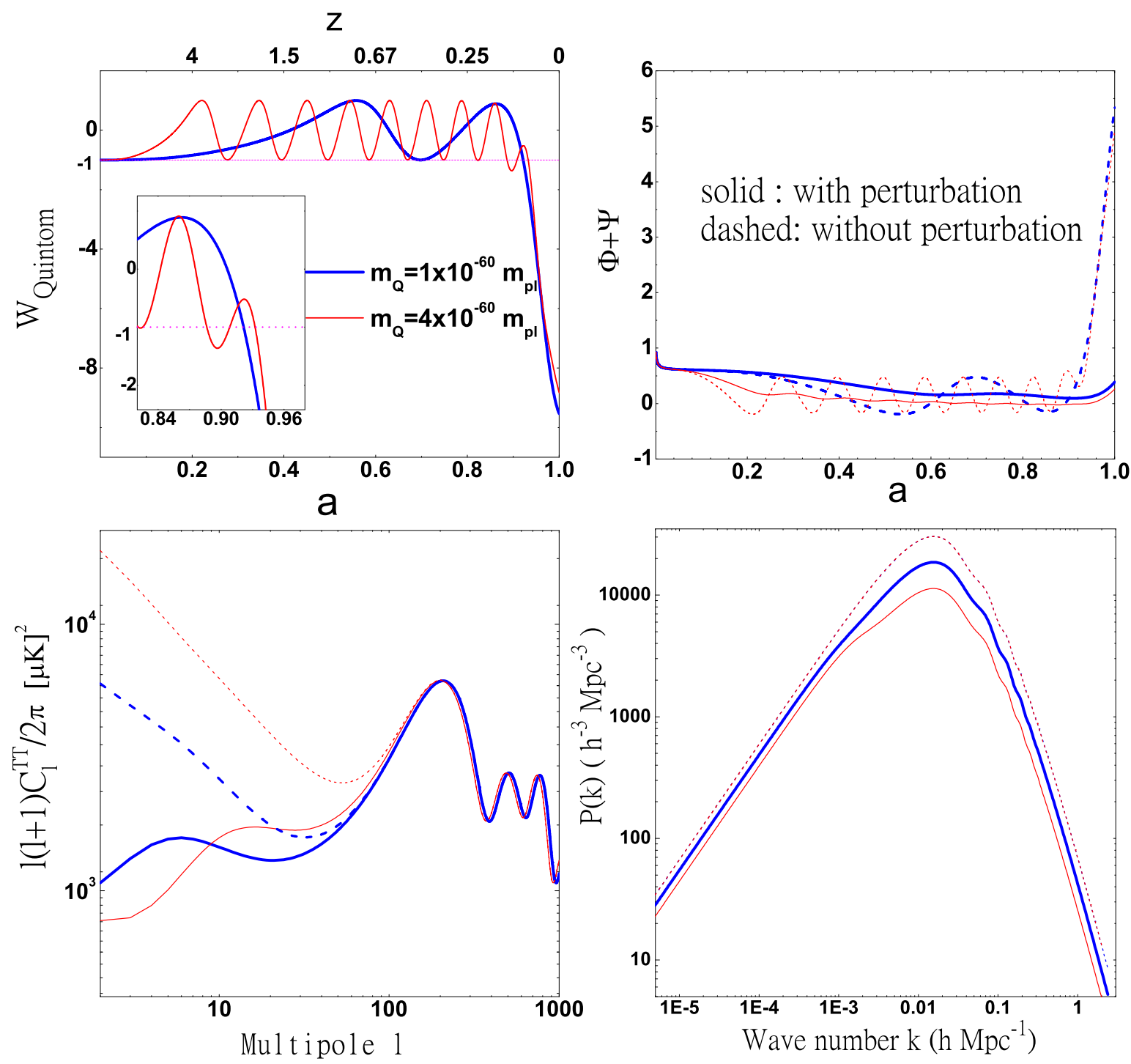

FIG. 1: Effects of the two-field oscillating quintom on the observables. The mass of the phantom field is fixed at $2.0 \times 10^{-60} m_{p l}$ and the mass of the quintessence field are $10^{-60} m_{p l}$ (thicker line) and $4.0 \times 10^{-60} m_{p l}$ (thinner line) respectively. The upper right panel illustrates the evolution of the metric perturbations $\Phi+\Psi$ of the two models when with(solid lines) and without(dashed lines) dark energy perturbations. The scale is $k \sim 10^{-3} \mathrm{Mpc}^{-1}$. The lower left panel shows the CMB effects and the lower right panel delineates the effects on the matter power spectrum when with(solid lines) and without(dashed lines) dark energy perturbations. 

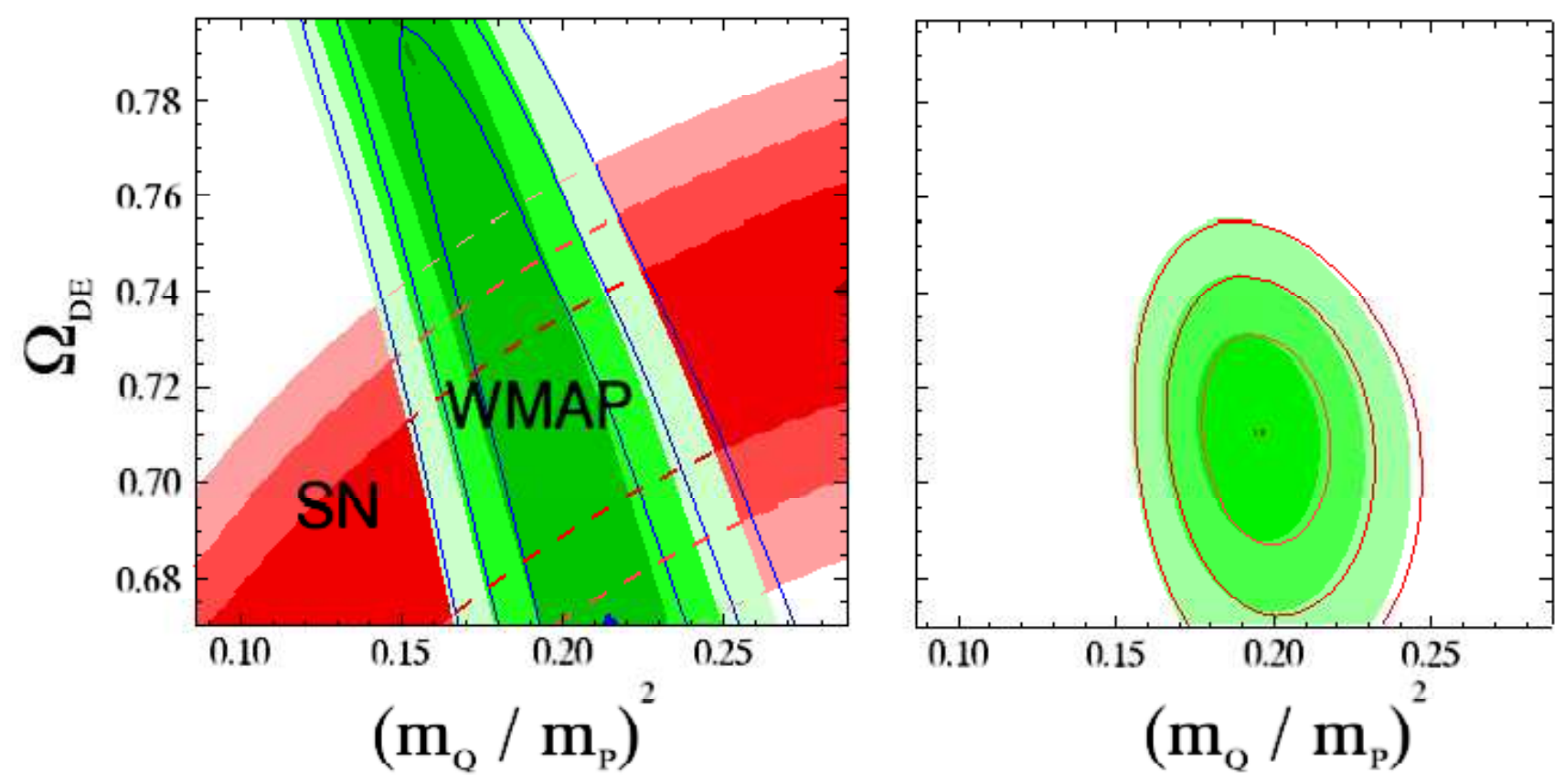

FIG. 2: $3 \sigma$ WMAP and SN constraints on two-field quintom model shown together with the best fit values. $m_{Q}$ and $m_{P}$ stand for the mass of quintessence and phantom respectively. We have fixed $m_{P} \sim 6.2 \times 10^{-61} m_{p l}$ and varied the value of $m_{Q}$. Left panel: Separate WMAP and SN constraints. The green(shaded) area is WMAP constraints on models where dark energy perturbations have been included and the blue area(contour with solid lines) is without dark energy perturbations. Right panel: Combined WMAP and SN constraints on the two-field quintom model with perturbations(green/shaded region) and without perturbations(red region/contour with solid lines). 

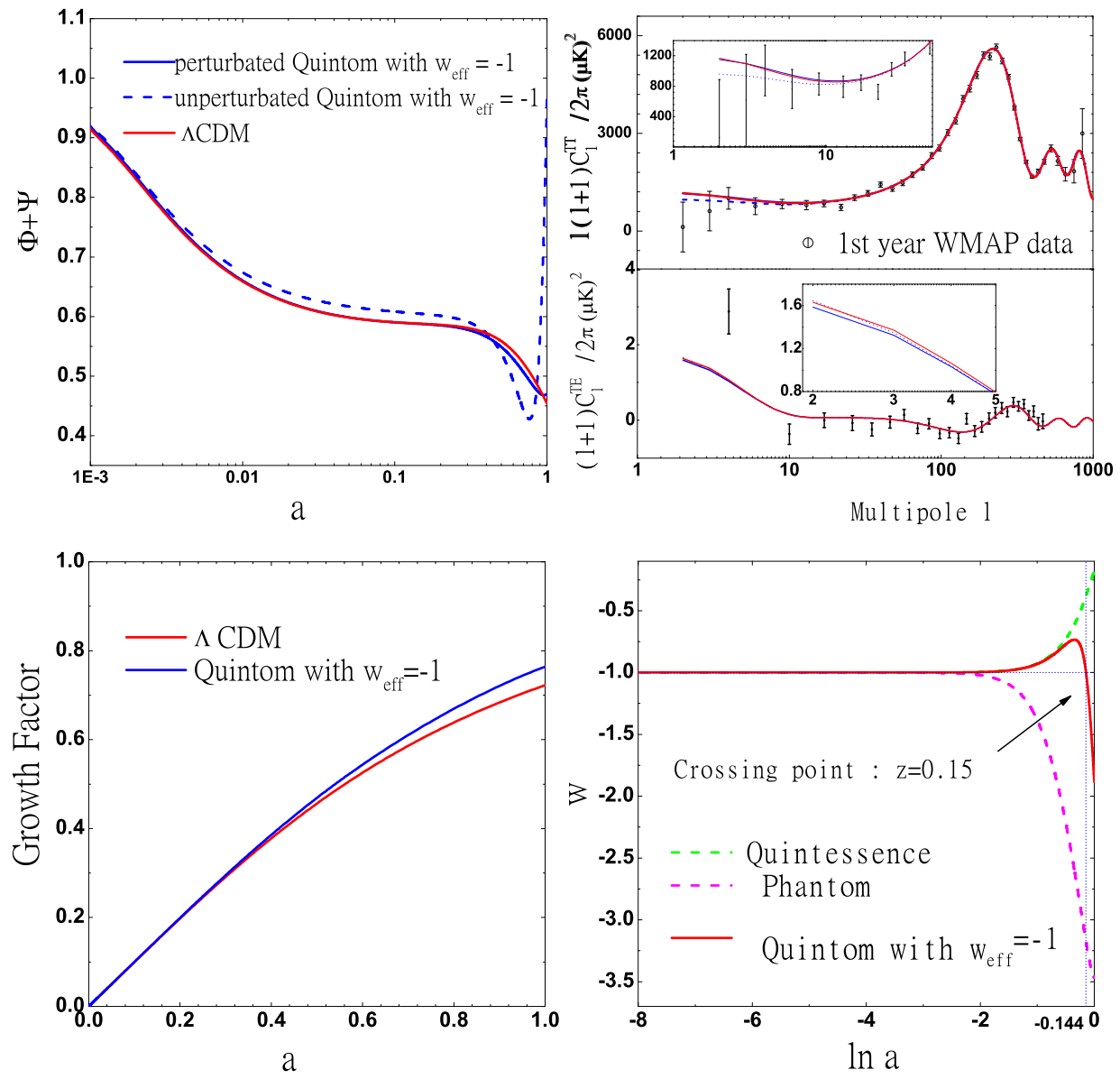

FIG. 3: Effects of the two-field quintom model where $w_{\text {eff }}=-1$ compared with cosmological constant in CMB(WMAP), the metric perturbations $\Phi+\Psi$ (the scale is $k \sim 10^{-3} \mathrm{Mpc}^{-1}$ ) and the linear growth factor. The binned error bars in the upper right panel are WMAP TT and TE data [4]. 


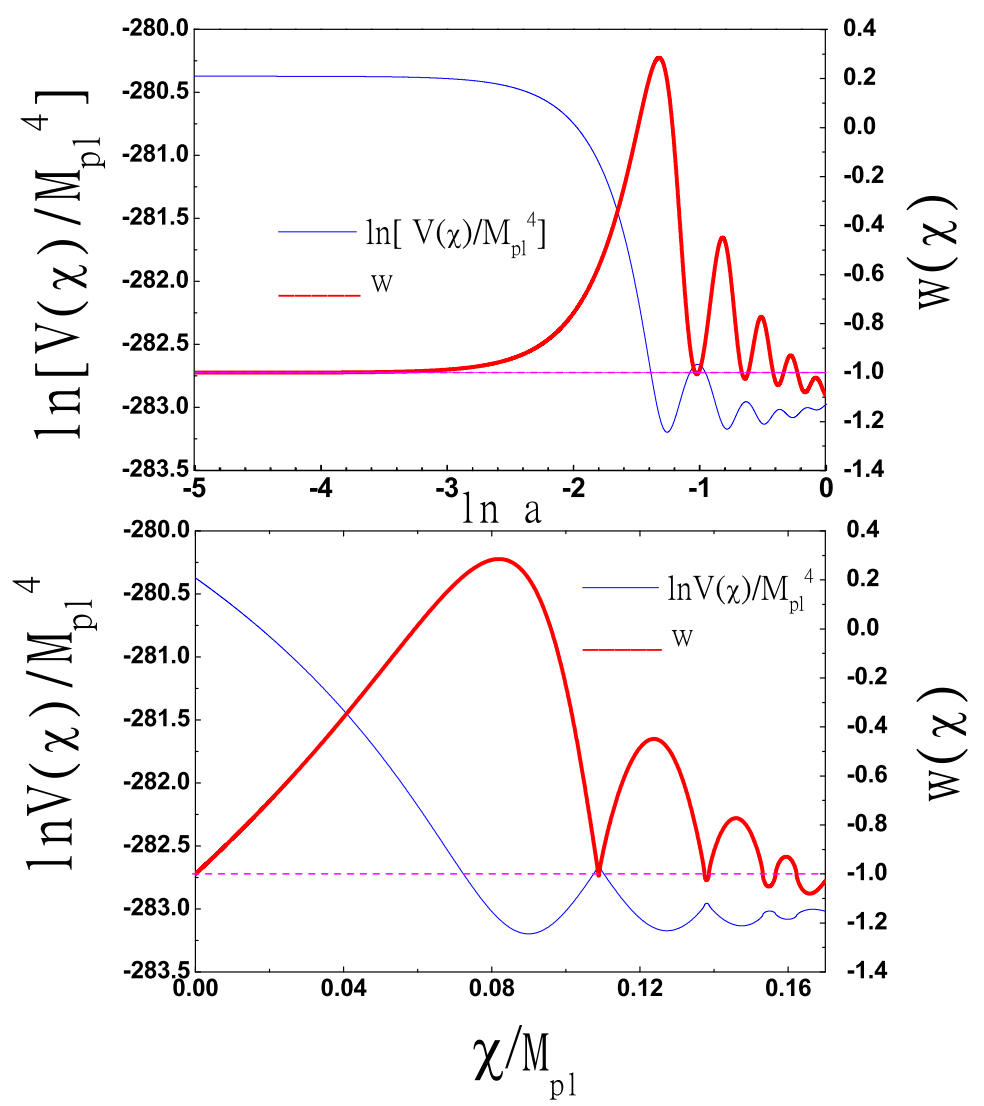

FIG. 4: Reconstructions of the effective adiabatic single field $\chi$ in the framwork of oscillating quintom. The background parameters have been chosen as $m_{\phi 1}=2 \times 10^{-60} m_{p l}, m_{\phi 2}=10^{-61} m_{p l}$, initial values are $\phi_{1 i}=0.09 m_{p l}, \phi_{2 i}=0.45 m_{p l}$ and $\dot{\phi_{1 i}}=\dot{\phi_{2 i}}=0$ early in radiation domination epoch and for this example we have $\Omega_{\phi 1}=0.2, \Omega_{\phi 2} \sim 0.54, h \sim 0.68$. The red lines are the total EOS of dark energy and the blue lines are the total potential. The dashed lines show the cosmological constant boundary. The upper panel delineates the late evolutions of the EOS and potential of dark energy and the lower panel shows the reconstructed values of $\chi$ and its potential, $\chi$ is a quintessence/phantom scalar when $w$ is above/below the dashed line. See the text for details. 

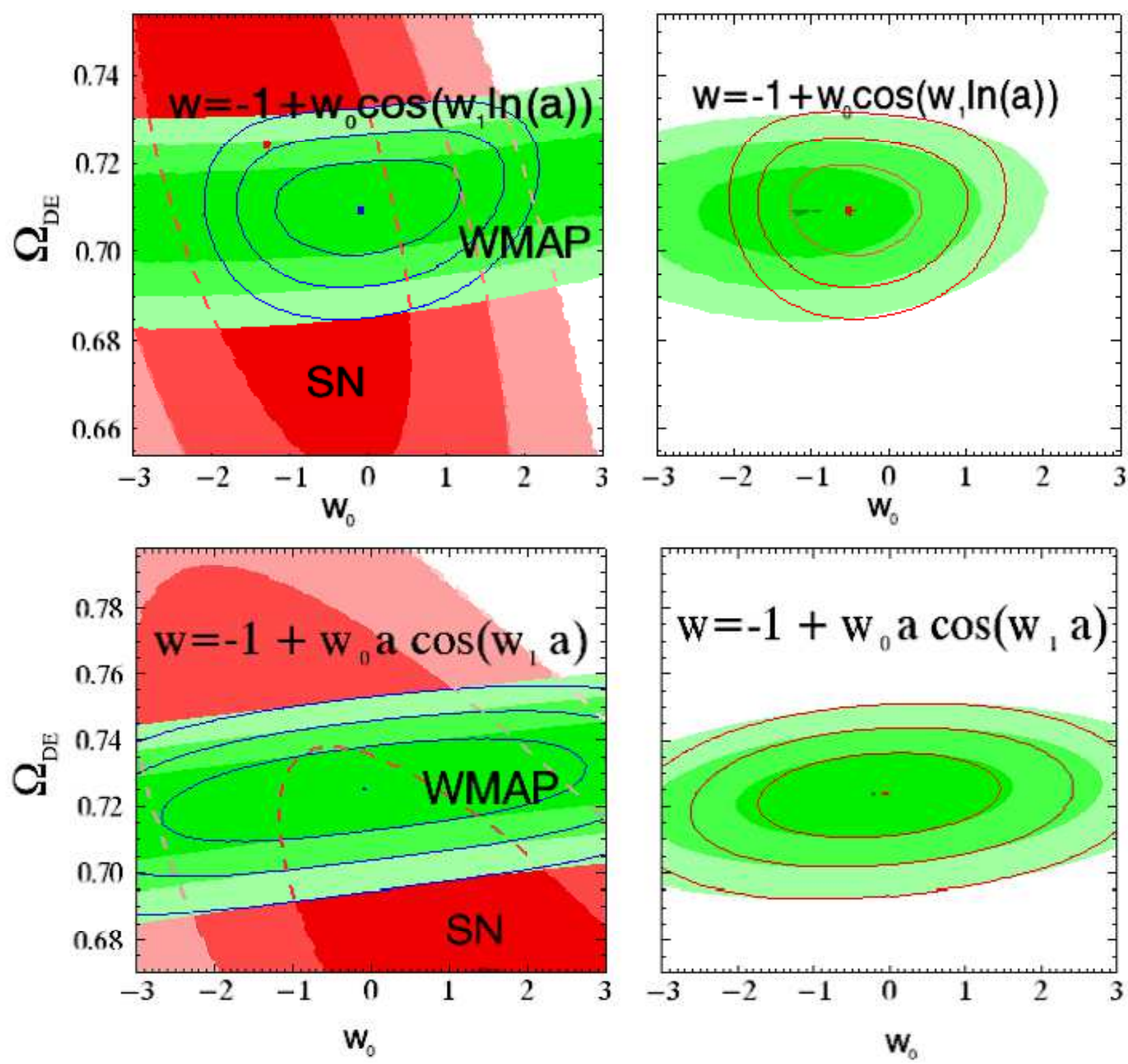

FIG. 5: $3 \sigma$ WMAP and SN constraints on the parametrized quintom models $w=-1+w_{0} \cos \left(w_{1} \ln a\right)$ and $w=-1+$ $w_{0} a \cos \left(w_{1} a\right)$, shown together with the best fit values. On the left panels the green(shaded) areas are WMAP constraints on models where dark energy perturbations have been included and the blue areas(contours with solid lines) are without dark energy perturbations. On the right panels models with perturbations are delineated in green(shaded) regions and the red regions(contour with solid lines) without perturbations. For illustrations we have fixed $w_{1}=20$ in the upper panels and $w_{1}=50$ in the lower panels. 


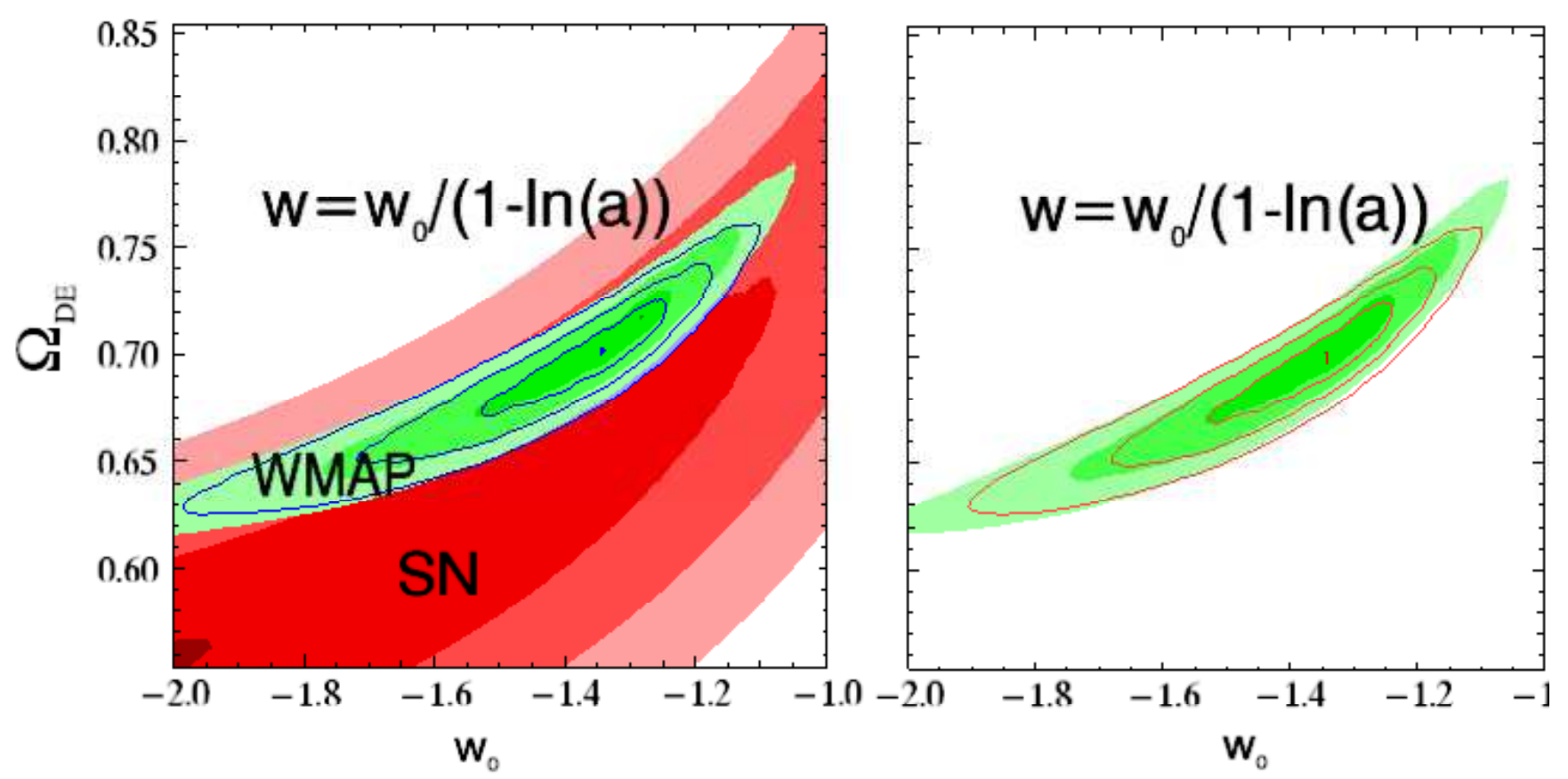

FIG. 6: $3 \sigma$ WMAP and SN constraints on the parametrized quintom model $w=w_{0} /(1-\ln a)$ shown together with the best fit values. Left panel: Separate WMAP and SN constraints. The green(shaded) area is WMAP constraints on models where dark energy perturbations have been included and the blue area( contour with solid lines) is without dark energy perturbations. Right panel: Combined WMAP and SN constraints on the parametrized quintom model with perturbations(green/shaded region) and without perturbations(red region/contour with solid lines). 